\title{
DAMAGE IDENTIFICATION OF BRIDGE SYSTEM BASED ON A HYBRID ALGORITHM
}

\author{
Jingzhou Xin, ${ }^{*}$ Hong Zhang, ${ }^{*}$ Jianting Zhou, ${ }^{*}$ Xiaogang $\mathrm{Li},{ }^{* *}$ and $\mathrm{Hu} \mathrm{Ma}{ }^{* * *}$
}

\begin{abstract}
Structural damage identification is fundamentally important for ensuring bridge safety and improving the pertinence of maintenance work. This paper proposes a new hybrid method for structural damage identification, which integrates the stepwise regression model based on uniform design and the intelligent optimization algorithm based on chaotic search. A numerical example is studied to investigate the efficiency and correctness of the proposed method. The simulation results demonstrated that the hybrid model can accurately identify the damage location and degree of the structure, the identification accuracy of the proposed method is satisfactory with $7.332 \%$ of mean relative percentage error and $9.878 \%$ of maximum relative percentage error. This paper provides a new way for damage identification of bridge structures.
\end{abstract}

\section{Key Words}

Damage identification, hybrid algorithm, chaotic search, bridge system

\section{Introduction}

With the development of information technology, such as sensing, computer, communication and data processing, and health monitoring systems are widely used in the bridge structures, which play a crucial role in relieving the increasing pressure on bridge safety [1], [2]. How to identify the bridge damage from the massive data obtained from the monitoring system and to predict the structural behaviour has become a common concern of the scientific and engineering communities [3].

For the structural damage identification, many studies have been conducted, which can be broadly classified into two categories. One is the deterministic method [4], including (i) static and dynamic fingerprint methods based on natural frequency and static displacement [5], modal

* School of Civil Engineering, Chongqing Jiaotong University, Chongqing, China; e-mail: xinjz@mails.cqjtu.edu.cn, \{hongzhang, jtzhou\}@cqjtu.edu.cn

** T.Y. Lin International Engineering Consulting (China) Co., Ltd., Chongqing, China; e-mail: li.xiaogang@tylin.com.cn

*** Chongqing Rail Transit (Group) Co., Ltd., Chongqing, China; e-mail: 609298395@qq.com

Corresponding author: Jianting Zhou

Recommended by Prof. Anmin Zhu

(DOI: 10.2316/J.2019.206-0144) flexibility-curvature [6], and modal strain energy [7], (ii) model updating methods [8], (iii) statistical signal processing methods, e.g., time series method [9], wavelet analysis method [10], and Hilbert-Huang transform method [11], and (iv) neural network (NN) method [12], etc. The second type is the uncertainty method [4], including damage identification using batch Bayesian estimation [13] and damage identification based on random finite element [14]. Every category of methods has their own advantages and disadvantages. For example, dynamic fingerprint methods are easy to implement, but many dynamic fingerprints are not sensitive to minor damage and difficult to perceive the damage degree [4]. The advantage of the statistical signal processing approach is that it does not require any elaborate finite element modelling. Such an approach is particularly suited for wireless analysis, which is capable of processing data at the sensor unit location through embedded algorithms [15], however, it allows only for the lowest level of damage detection, i.e., deciding whether the damage has occurred or not [16]. NN has a strong nonlinear mapping function and fault tolerance performance, however, it is arduous to determine the key parameters and avoid the subjectivity caused by artificial selection [17]. Uncertain damage identification methods can cope with the uncertainty caused by noise interference, measurement error, and finite element simulation approximation. But the Bayesian method is generally unable to solve large-scale computational problems with unknown parameters due to the limitation of dimension. The random finite element method is divorced from the measured signal, and it has the problem of enormous computing.

As damage identification method based on model updating can realize damage location and quantification by directly utilizing ambient excitation without interrupting traffic, which meets the requirements of online health monitoring, it gets a growing concern in the recent years. Finite element model (FEM) updating method can be divided into two categories, namely, matrix method and design parameters method, the latter takes the structural parameters as updating targets, which is of higher practical value in projects because of its definite physical meaning. From a mathematical view, by transferring the local damage identification problem to the objective function optimization, the damage can be obtained by minimizing the objective function. And the objective function is formulated of the difference between the calculated and the measured data 
from the certain structural system. To solve the optimization problems, intelligent algorithms are widely introduced [18], [19]. But these swarm intelligence techniques both have common failing on slow convergence, and some of them may fall into local optimization easily [20].

Apart from the existing intelligent algorithms, monkey algorithm (MA), initially proposed by Zhao and Tang in 2008 [21], is an intelligent optimization algorithm that was derived from the simulation of mountain-climbing processes of monkeys. The optimal solution search is realized by simulating the action of climbing, watching, jumping, and somersaulting in the monkey mountain climbing process. Due to the merits of simple structure, strong global optimization ability, dimension insensitivity to the optimization problems, few parameters, and easiness to implement, MA is widely used in the field of optimal sensor placement [22], hybrid power systems optimization [23], and transmission network expansion planning [24], but rarely reported on the application of damage identification.

The present work is concerned with the formulation of a damage identification approach built on the response surface method (RSM) and the chaotic monkey algorithm (CMA). First, sample points are obtained by the experimental design and the FEMs, and the approximate analytical expressions between structural stiffness and structural response indexes are obtained by using RSM. Second, the damage identification is accomplished by solving objective function using CMA, with the aim at determining the stiffness of damaged structure that minimizes the difference between the experimentally obtained response and the corresponding vector predicted by an RSM of the structure. The flowchart of the proposed method is shown in Fig. 1.

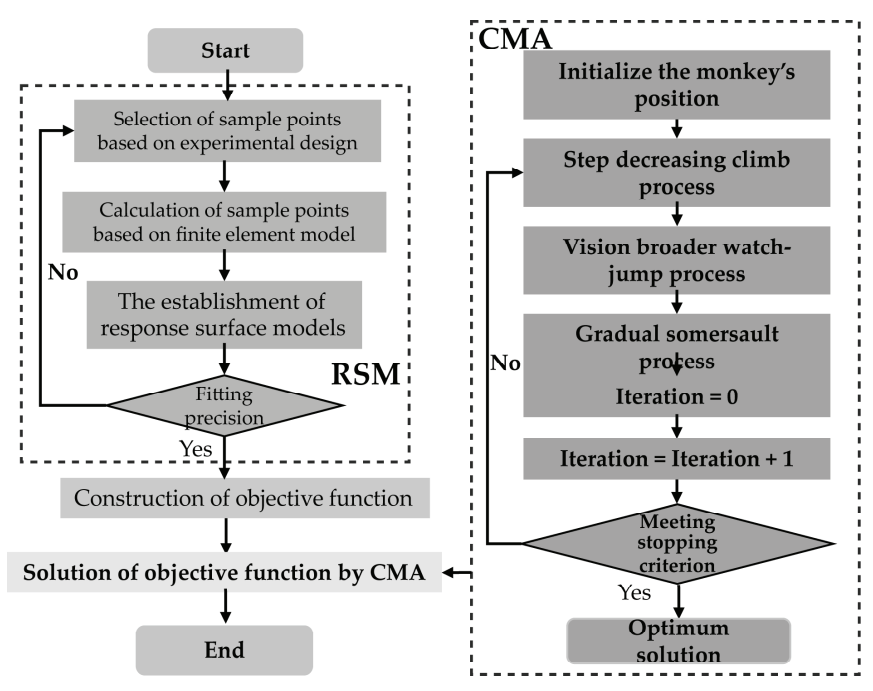

Figure 1. Flowchart of the proposed hybrid method.

\section{Chaotic Monkey Algorithm}

MA mainly consists of three processes, i.e., the climbing process, the watch-jump process, and the somersault process. The climb process is designed to search the local optima, the watch-jump process accelerates the search process by looking for a better solution than the current location in the vicinity, and the somersault process is employed to make the monkeys transfer to other search domains subtly to avoid plunging into the local optimum.

CMA [25] is an improved intelligence algorithm combining MA with a chaotic search. As chaos description is a nonlinear state which has deterministic, randomness, and ergodicity, this improvement can effectively reduce the risk of MA falling into a local optimum. CMA mainly consists of the following four steps: chaos initialization, step decreasing climb process, vision broader watch-jump process, and gradual somersault process.

\subsection{Chaos Initialization}

Assume $M$ monkeys make up a group. The position of a monkey can be expressed by the vector $X_{i}=\left(x_{i 1}, x_{i 2}, \ldots, x_{i n}\right)$, where $i=1-M$, and $n$ is the solution vector dimension, which is equal to the number of parameters to be modified in this work. $N$-dimensional chaotic variable can be generated by Logistic mapping by

$$
y^{(i t e r+1)}=4 y^{(i t e r)}\left(1-y^{(i t e r)}\right)
$$

where $y^{(i t e r)}$ is a chaotic variable, iter represent iteration times; $y^{(i t e r)} \in(0,1), y^{(0)} \notin\{0,0.25,0.75,1\}$. The range of chaotic variables can be converted to the domain of objective function optimization problem by

$$
x^{(i t e r+1)}=a+y^{(i t e r+1)}(b-a)
$$

where $a$ and $b$ are the upper and lower limit of the domain, respectively.

\subsection{Step Decreasing Climb Process}

The climb process is a vital step to realize gradual optimization through iteration, which was designed to calculate the pseudo gradient to reach higher optimization speed. The outline of the climb process can be described as:

Step 1. Generate increment $\Delta \boldsymbol{X}_{i}$ randomly, $\Delta \boldsymbol{X}_{i}=$ $\left(\Delta x_{i 1}, \Delta x_{i 2}, \ldots, \Delta x_{i n}\right), \quad j=1,2, \ldots, n, \quad \Delta x_{i j}=\alpha \quad$ or $\Delta x_{i j}=-\alpha$ with the same probability, $\alpha$ is the step length of climb process, $\alpha>0$.

Step 2. Calculate $f_{i j}^{\prime}\left(x_{i}\right)=\frac{f\left(x_{i}+\Delta x_{i}\right)-f\left(x_{i}-\Delta x_{i}\right)}{2 \Delta x_{i j}}$, the vector $\left(f_{i 1}^{\prime}\left(\boldsymbol{X}_{i}\right), f_{i 2}^{\prime}\left(\boldsymbol{X}_{i}\right), \ldots, f_{i n}^{\prime}\left(\boldsymbol{X}_{i}\right)\right)$ is the pseudo gradient of the objective function at the position $\boldsymbol{X}_{i}$.

Step 3. Compute $y_{j}=x_{i j}+\alpha \cdot \operatorname{sign}\left(f_{i j}^{\prime}\left(x_{i}\right)\right), \boldsymbol{y}=\left(y_{1}\right.$, $\left.y_{2}, \ldots, y_{n}\right)$, and update the vector position when $y$ is in the definition domain of the objective function, otherwise the original position remains unchanged.

Step 4. Repeat Steps 1-3 until the value of the objective function between two adjacent iterative processes has no significant change, or achieve the maximum number of iterations.

Here, $\alpha$ plays a crucially important role. Generally, there is a positive correlation between the accuracy and 
the step length, and a negative correlation between the computing time and $\alpha$. In this paper, the balance between the computation time and the accuracy of the solution is realized by

$$
\alpha^{(k+1)}=\theta \cdot \alpha^{(k)}
$$

where $\theta$ is the decreasing factor of step length, $0<\theta<1 ; k$ is the number the monkey climbs.

\subsection{Vision Broader Watch-Jump Process}

Each monkey has reached the top of the mountain after the climb process, i.e., the objective function achieves the local optimal. Then, the watch-jump process is designed to make monkeys look around to observe whether there is a better position in the surrounding area. Steps are as follows:

Step 1. Generate real numbers $y_{i}$ in the field of vision $\left(x_{i j}-\beta, x_{i j}+\beta\right)$ randomly, $\beta$ denotes the maximum distance that the monkey can see (termed as the vision of the monkey); $\beta>0$, set $y=\left(y_{1}, y_{2}, \ldots, y_{n}\right)$.

Step 2. Compute $f(\boldsymbol{y})$, and update monkey's position from $\boldsymbol{X}_{i}$ to $\boldsymbol{y}$ when $f(\boldsymbol{y})$ is superior to $f\left(\boldsymbol{X}_{i}\right)$ and $\boldsymbol{y}$ meet the constraints, otherwise, repeat Step 1 until finding the appropriate one.

Step 3. Carry out the climb process with $\boldsymbol{y}$ as the initial position.

As the search process goes on, the monkeys climb to higher peaks, and their vision should also increase. The gradual increase of vision length is designed in CMA:

$$
\beta^{(i t e r+1)}=\rho \cdot \beta^{(i t e r)}
$$

where $\rho$ is the growth factor of vision length.

Higher mountain peak and wider horizon also mean more time to search for better locations. Here, watching time $\omega$ is defined as follows:

$$
\omega^{(i t e r+1)}=\varphi \cdot \omega^{(i t e r)}
$$

where $\varphi$ is the growth factor of watching time.

\subsection{Gradual Somersault Process}

The somersault process is executed to make the search progress transfer to a new domain. The somersault movement needs a fulcrum $P_{j}$, which is depicted by (6):

$$
P_{j}=\frac{1}{M-1}\left(\sum_{l=1}^{M} x_{l j}-x_{i j}\right)
$$

Each monkey somersaults to the new search domain along the direction from the current position to the fulcrum by

$$
x_{i j}^{(i t e r+1)}=P_{j}+\beta\left|P_{j}-x_{i j}^{i t e r}\right|
$$

where $\beta$ constraints the distance that monkey can somersault, as $\beta$ increases progressively, the distance that monkeys can somersault grows, it is also an improvement to avoid falling into local optimum in CMA.

\section{Construction of Objective Function Based on Response Surface Method}

Here, the damage state of the structure is continuously described and spatially discretized by the FEM, and the RSM is considered for providing approximate relations between the structural parameters and the structural response. Therefore, the corresponding inverse problem of damage identification is defined as a minimization one, where the aim is to find the parameters that minimize the difference between the experimental response vector, composed with the response obtained from a modal test on the supposed damaged structure [26]. The objective function under certain constraint conditions can be constructed by

$$
\left\{\begin{array}{l}
\operatorname{Min}\|f(\boldsymbol{p})\|^{2}, f(\boldsymbol{p})=\left\{f_{\mathrm{RSM}}\right\}-\left\{f_{i}\right\} \\
V L B \leq \boldsymbol{p} \leq V U B
\end{array}\right.
$$

where $\boldsymbol{p}$ represents structural parameters to be identified, as the stiffness of the damaged element is reduced when a damage event occurs, the damage of element was simulated via the reduction of the elasticity modulus in this paper; $f_{\mathrm{RSM}}$ and $f_{i}$ denote the frequency predicted by the RSM and actual structural response obtained from a modal test on the supposed damaged structure; $V U B$ and $V L B$ are the upper and lower bounds of the structural parameters, respectively.

In the next subsection, the procedure of RSM is interpreted.

\subsection{Basic Function Selection of Response Surface}

In this study, the second-order polynomial model without interaction terms is used as the function form of the response surface model:

$$
R=a+\sum_{i=1}^{n^{\prime}} b_{i} x_{i}+\sum_{i=1}^{n^{\prime}} c_{i} x_{i}^{2}
$$

where $R$ represents the structural response of the sample point obtained by FEM, $x_{i}$ and $x_{i}^{2}$ are the structural parameters that represent structural stiffness; $n^{\prime}$ is the number of structural parameters.

The number and distribution of sample points are vital factors, which affect the efficiency and accuracy of the response surface model. On the one hand, small samples lead to spurious relationship between structural response and parameters. On the other hand, more samples improve the fitting accuracy but reduce the efficiency. To meet this problem, the uniform design based on pseudo-Monte Carlo method is adapted to determine the distribution and quantity of samples [27]. 


\subsection{Establishment of Response Surface Model}

Equation (9) can be expressed as $\mathbf{X B}=\mathbf{R}$, with

$$
\begin{aligned}
& \mathbf{X}=\left[\begin{array}{ccccccccc}
1 & x_{11} & x_{12} & \cdots & x_{1 n^{\prime}} & x_{11}^{2} & x_{12}^{2} & \cdots & x_{1 n^{\prime}}^{2} \\
1 & x_{21} & x_{22} & \cdots & x_{2 n^{\prime}} & x_{21}^{2} & x_{22}^{2} & \cdots & x_{2 n^{\prime}}^{2} \\
\vdots & \vdots & \vdots & \ddots & \vdots & \vdots & \vdots & \vdots & \vdots \\
1 & x_{m 1} & x_{m 2} & \cdots & x_{m n^{\prime}} & x_{m 1}^{2} & x_{m 2}^{2} & \cdots & x_{m n^{\prime}}^{2}
\end{array}\right] \\
& \mathbf{B}=\left(\begin{array}{lllllll}
a & b_{i} & \cdots & b_{n^{\prime}} & c_{i} & \cdots & c_{n^{\prime}}
\end{array}\right)^{\mathrm{T}} \\
& \mathbf{R}=\left(\begin{array}{llll}
R_{1} & R_{2} & \cdots & R_{m}
\end{array}\right)^{\mathrm{T}}
\end{aligned}
$$

where $m$ is the number of sample points.

An unbiased estimate $\mathbf{B}$ for the coefficients may be obtained from the least square method as

$$
\mathbf{B}=\left(\mathbf{X}^{\mathrm{T}} \mathbf{X}\right)^{-1} \mathbf{X}^{\mathrm{T}} \mathbf{R}
$$

The significance of each parameter is evaluated according to $F$-test. To determine the significance of the item $g+1$ to the regression model, assuming that the regression model contains $g$ term, statistic $F_{g+1}$ is calculated by

$$
F_{g+1}=\frac{\left(S S E_{g}-S S E_{g+1}\right) /\left(v_{g}-v_{g+1}\right)}{S S E_{g+1} / v_{g+1}}
$$

where $S S E_{g}$ is the sum of square error of the response surface model contains $g$ items and response surface model contains $g+1$ items, respectively; and $v_{g}$ and $v_{g+1}$ are the freedom of above two response surface model, respectively. At the significance level $\chi$, when the statistic $F_{g+1}>F_{\chi}(1, m-p-2)$, item $g+1$ is significant, the regression model should include this item.

\subsection{Accuracy Test of Response Surface Model}

Essentially, the response surface model is an approximate estimate of the implicit relationship between random variables and structural responses. Analysis of variance is necessary for checking the relationship between response and variables in statistical significance. In this paper, the accuracy of the response surface model is tested by the modified coefficient of determination $R_{a d j}^{2}$ and $F$-test.

The closer $R_{a d j}^{2}$ is to 1 , the higher the accuracy of the response surface model achieves.

$$
\begin{gathered}
R_{a d j}^{2}=1-\frac{S S E /(m-p-1)}{S S T /(m-1)} \\
S S T=\sum_{i=1}^{m}\left(R_{i}-\bar{R}\right)^{2} \\
S S E=\sum_{i=1}^{m}\left(R_{i}-\widehat{R}_{i}\right)^{2}
\end{gathered}
$$

where $S S T$ is the residual sum of squares of $R$, which reflects the fluctuation of $R ; S S E$ is the sum of square error of $R$, which reflects the effect of the error on response fluctuation; $\bar{R}$ is the mean value, $\bar{R}=\frac{1}{m} \sum_{i=1}^{m} R_{i} ; R_{i}$ and $\widehat{R}_{i}$ are the finite element response values and RSM values, respectively.

At the significance level $\chi$, the response surface model is significant when the statistic $F>F_{\chi}(p, m-p-1)$. The statistic $F$ of response surface model can be calculated by

$$
\begin{gathered}
F=\frac{S S R / p}{S S E /(m-p-1)} \\
S S R=\sum_{i=1}^{m}\left(\widehat{R}_{i}-\bar{R}_{i}\right)^{2}
\end{gathered}
$$

where $S S R$ is the regression sum of squares of $R ; p$ is the number of non-constant terms in the response surface function.

\section{Numerical Example}

In this work, a simply supported beam shown in Fig. 2 is taken as an example to verify the effectiveness of the proposed federated method. The concrete beam has a length of $6 \mathrm{~m}$ with a rectangular cross-section whose size is $0.25 \mathrm{~m}$ in the width direction and $0.2 \mathrm{~m}$ in the height direction. Spatial beam element is selected to set up the numerical model which is divided into ten parts. The density and elasticity modulus are $2,500 \mathrm{~kg} / \mathrm{m}^{3}$ and $3.2 \times 10^{4} \mathrm{MPa}$, respectively. Poisson's ratio is 0.2 .

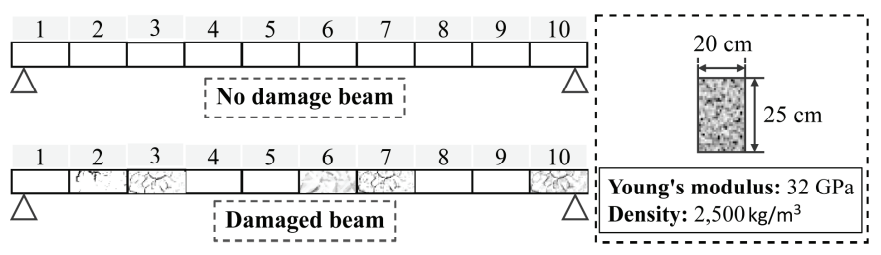

Figure 2. Schematic diagram of numerical models.

Here, two scenarios are set as follows:

Scenario 1: No damage beam.

Scenario 2: $9 \%$ damage in element 2, $23 \%$ damage in element 3,18\% damage in element $6,15 \%$ damage in element 7 , and $21 \%$ damage in element 10 .

In this research, the stable global variable frequency, which has relatively high measurement precision is chosen as the target to be modified. The first five frequencies of the two models are listed in Table 1. As shown in Table 1, there is a positive correlation between the damage and the structural natural frequency.

In this study, the stiffness reduction factor $z_{i}$ (termed as reduction of elastic modulus of finite element $i$ ) is introduced, $z_{i} \in[0.7,1.0]$. Sample points with ten parameters and twenty-three levels are obtained by uniform design [27]. The stiffness reduction factor $z_{i}$ at the sample points 
Table 1

Natural Frequencies of Structures between the No Damage Beam and Damaged Beam

\begin{tabular}{|l|c|c|c|c|c|}
\hline $\begin{array}{l}\text { Mode/Frequency } \\
\text { (Hz) }\end{array}$ & 1 & 2 & 3 & 4 & 5 \\
\hline $\begin{array}{l}\text { No damage } \\
\text { beam (1) }\end{array}$ & 8.982 & 25.225 & 35.779 & 68.640 & 79.884 \\
\hline Damaged beam (2) & 8.541 & 23.925 & 34.024 & 65.092 & 76.355 \\
\hline $\begin{array}{l}\text { Relative error }= \\
\text { (1)-(2)/(1)(\%) }\end{array}$ & 4.910 & 5.154 & 4.905 & 5.170 & 4.418 \\
\hline
\end{tabular}

and the corresponding structure responses obtained from FEMs are shown in Table 2.

Five second-order polynomial without cross terms response surface models are obtained based on the stepwise regression using sample data from Table 2, the explicit expressions of the implicit relationship between the structural frequencies and the stiffness reduction coefficients of the structural elements are realized.

$$
\left\{\begin{array}{c}
f_{1}=5.492+0.769 z_{4}+0.512 z_{8}+0.234 z_{9}+0.121 z_{2}^{2} \\
\quad+0.288 z_{3}^{2}+0.576 z_{5}^{2}+0.567 z_{6}^{2}+0.445 z_{7}^{2} \\
f_{2}=14.895+3.353 z_{1}+0.967 z_{4}+0.875 z_{7}+0.723 z_{9} \\
\quad+0.429 z_{2}^{2}+1.068 z_{5}^{2}+1.033 z_{6}^{2}+1.911 z_{10}^{2} \\
f_{3}=18.120+3.734 z_{3}+2.658 z_{4}+2.522 z_{7}+3.699 z_{8} \\
\quad+2.408 z_{9}+0.228 z_{1}^{2}+1.427 z_{2}^{2}+0.362 z_{5}^{2}+0.276 z_{6}^{2} \\
+0.366 z_{10}^{2} \\
f_{4}=31.971+14.125 z_{1}+4.675 z_{3}+5.393 z_{4}+4.974 z_{8} \\
\quad+6.493 z_{10}-4.857 z_{1}^{2}+0.544 z_{2}^{2}+0.943 z_{5}^{2}+0.844 z_{6}^{2} \\
\quad+3.069 z_{7}^{2}+0.516 z_{9}^{2} \\
f_{5}=33.884+7.603 z_{2}+1.239 z_{4}+6.197 z_{6}+4.087 z_{8} \\
+23.933 z_{9}+1.455 z_{1}^{2}+2.587 z_{3}^{2}+0.608 z_{7}^{2}+1.544 z_{10}^{2}
\end{array}\right.
$$

Table 2

Sample Points

\begin{tabular}{|c|c|c|c|c|c|c|c|c|c|c|c|c|c|c|c|}
\hline \multirow[t]{2}{*}{ FEM } & \multicolumn{10}{|c|}{$z_{i}$} & \multicolumn{5}{|c|}{ Mode/Frequency (Hz) } \\
\hline & $Z_{1}$ & $Z_{2}$ & $Z_{3}$ & $Z_{4}$ & $Z_{5}$ & $Z_{6}$ & $Z_{7}$ & $Z_{8}$ & $Z_{9}$ & $Z_{10}$ & 1 & 2 & 3 & 4 & 5 \\
\hline 1 & 0.805 & 0.985 & 0.985 & 0.865 & 0.790 & 0.745 & 0.775 & 0.835 & 0.760 & 0.820 & 8.111 & 22.654 & 33.147 & 62.740 & 72.974 \\
\hline 2 & 850 & 790 & 000 & 940 & 0.985 & & .970 & 0.925 & & & & 23.840 & 34.407 & 65.804 & 75.772 \\
\hline 3 & 955 & .745 & .880 & 0.955 & 0.730 & 0.850 & 0.700 & 0.790 & 0.745 & 0.835 & 8.029 & 23.027 & 32.088 & 62.851 & 71.037 \\
\hline 4 & 000 & 835 & 865 & 0.835 & 0.880 & 0.940 & 0.730 & 0.895 & 0.985 & 1.000 & 8.324 & 24.334 & 33.250 & 4.747 & 75.913 \\
\hline 5 & 940 & 000 & .775 & 0.790 & 1.000 & 0.910 & 0.820 & 0.970 & 0.805 & 0.850 & 8.414 & 3.866 & 33.171 & 3.781 & 75.673 \\
\hline 6 & 865 & 0.805 & .700 & 0.910 & 0.895 & 0.955 & 0.790 & 0.715 & 0.775 & 0.775 & 8.207 & 23.151 & 31.515 & 61.400 & 72.140 \\
\hline 7 & 700 & 0.820 & 0.850 & 0.820 & 0.715 & 0.730 & 0.955 & 0.955 & 0.790 & 0.805 & 8.073 & 22.070 & 33.000 & 2.291 & 71.180 \\
\hline 8 & 925 & .775 & .910 & 0.700 & 0.925 & 0.700 & 0.805 & 0.850 & 0.925 & 0.790 & 8.035 & 22.879 & 32.541 & 62.268 & 72.913 \\
\hline 9 & 835 & 0.955 & 0.730 & 0.730 & 0.760 & 0.820 & 0.715 & 0.910 & 0.910 & 0.760 & 7.936 & 22.506 & 32.210 & 60.729 & 73.064 \\
\hline 10 & 820 & 0.715 & 0.940 & 0.745 & 0.820 & 0.985 & 0.850 & 0.940 & 0.715 & 0.880 & 8.282 & 23.110 & 32.560 & 63.287 & 72.357 \\
\hline 11 & 745 & 0.865 & 0.955 & 0.970 & 0.865 & 0.895 & 0.760 & 1.000 & 0.865 & 0.730 & 8.449 & 22.619 & 33.831 & 63.065 & 74.612 \\
\hline 12 & 0085 & 0.880 & 0.970 & 0.775 & 0.850 & & 1.000 & 0.745 & & 0.745 & & 23.266 & 33.004 & & \\
\hline 13 & 715 & 0.970 & 0.895 & 0.880 & 0.940 & 1.000 & 0.910 & 0.775 & 0.895 & 0.820 & 8.561 & 23.216 & 33.439 & 62.897 & 75.750 \\
\hline 14 & 0.760 & 0.850 & 0.745 & 0.715 & 0.910 & 0.865 & 0.940 & 0.805 & 0.730 & 0.985 & 8.179 & 23.250 & 31.944 & 62.388 & 72.365 \\
\hline 15 & 0.895 & 0.940 & 0.835 & 0.925 & 0.955 & 0.760 & 0.880 & 0.880 & 0.700 & 0.700 & & 22.899 & 32.975 & & 72.648 \\
\hline 16 & 0.910 & 0.910 & 0.805 & 0.985 & 0.745 & 0.970 & 0.985 & 0.865 & 0.835 & 0.955 & 8.449 & 23.998 & 33.640 & 65.472 & 74.325 \\
\hline 17 & 0.730 & 0.730 & 0.820 & 0.805 & 0.970 & 0.790 & 0.745 & 0.730 & 0.850 & 0.940 & & 22.945 & 31.671 & 1.386 & 71.917 \\
\hline 18 & 0.970 & 0.760 & 0.715 & 0.895 & 0.805 & 0.775 & 0.895 & 0.985 & 0.880 & 0.925 & 8.195 & 23.649 & 32.910 & 64.504 & 72.791 \\
\hline 19 & 0.880 & 0.925 & 0.925 & 0.760 & 0.700 & 0.880 & 0.865 & 0.700 & 0.940 & 0.910 & 8.059 & 23.301 & 32.684 & 62.810 & 73.568 \\
\hline 20 & 0.775 & 0.895 & 0.760 & 1.000 & 0.835 & 0.715 & 0.835 & 0.760 & 1.000 & 0.865 & 8.141 & 22.939 & 32.826 & 62.470 & 72.967 \\
\hline 21 & 790 & 0.700 & 0.790 & 0.850 & 0.775 & 0.925 & 0.925 & 0.820 & 0.970 & 0.715 & 8.251 & 22.548 & 32.536 & 61.846 & 72.188 \\
\hline
\end{tabular}


Table 3

Significance Test of Response Surface Models

\begin{tabular}{|l|r|r|r|r|r|}
\hline $\begin{array}{l}\text { Response } \\
\text { surface } \\
\text { models }\end{array}$ & $f_{1}$ & $f_{2}$ & $f_{3}$ & $f_{4}$ & \multicolumn{1}{c|}{$f_{5}$} \\
\hline$F$ & 529.627 & 289.216 & 499.100 & 533.711 & 361.630 \\
\hline$R_{a d j}^{2}$ & 0.995 & 0.991 & 0.996 & 0.997 & 0.995 \\
\hline
\end{tabular}

Table 4

Parameters Setting in CMA

\begin{tabular}{|l|c|c|c|c|c|c|c|c|}
\hline$M$ & $k$ & $\alpha$ & $\beta$ & $\omega$ & $\theta$ & $\rho$ & $\varphi$ & iter \\
\hline 3 & 10 & 0.01 & 0.03 & 20 & 0.95 & 1.05 & 1.05 & 90 \\
\hline
\end{tabular}

Under the significant level of 0.05 , the maximum value of the statistic $F$ is 3.11. As shown in Table 3, the statistic $F$ of the response surface models is much larger than the critical value, the response surface models are significant. The modified coefficient of determination $R_{a d j}^{2}$ shows that at least $99.1 \%$ of the total deviation of the five response surface models is caused by the variation of the test variables. To put it from another angle, only $0.9 \%$ of the total deviation of the responses cannot be explained by the model. The fitness of the models is satisfactory.

According to (8), the objective function used for damage detection can be expressed as

$$
\left\{\begin{array}{l}
\operatorname{Min} f\left(z_{1}, z_{2}, \ldots, z_{10}\right) \text { s.t. } z_{1}, z_{2}, \ldots, z_{10} \in[0.7,1.0] \\
f\left(z_{1}, z_{2}, \ldots, z_{10}\right)=\left(f_{1}-8.541\right)^{2}+\left(f_{2}-23.925\right)^{2} \\
+\left(f_{3}-34.024\right)^{2}+\left(f_{4}-65.092\right)^{2}+\left(f_{5}-76.355\right)^{2}
\end{array}\right.
$$

Solve the function (19) by using CMA, all parameters setting of CMA is shown in Table 4. Meanwhile, a classic nonlinear programming solver (NLP) is also implemented here using the MATLAB Optimization Toolbox. The results are shown in Table 5 .

In Table 5, RPE denotes relative percentage error; MRPE represents mean relative percentage error. As shown in Table 5, the identification accuracy of the CMA is satisfactory with $7.332 \%$ of MRPE and $9.878 \%$ of maximum RPE. Contrastively, the maximum RPE of the parameters identified by the NLP is $29.400 \%$, with an MRPE of $10.531 \%$. The CMA has obviously better identification performance.

The identified parameters are used to replace the initial values of the parameters for the FEM. The frequencies of the modified models are shown in Table 5 . As can be seen from Table 5, the maximum RPE of the response obtained from the model modified by CMA is only $0.527 \%$, with an MRPE of $0.200 \%$. Compared with the model modified by NLP, the proposed method has more advantages in structural response prediction.

\section{Conclusion}

In this paper, a hybrid damage identification method based on RSM-CMA is investigated. Some concluding remarks can be summarized as follows:

1. In the formulation of the inverse problem of damage identification, the FEM of the structure was replaced by the response surface model, it provided polynomial relations between the stiffness reduction factor structural responses, which is a computationally more efficient approach. Furthermore, the proposed method only needs the first few natural frequencies of the structure in the identification.

2. The CMA provides a feasible method for the structural damage identification based on model updating.

Table 5

Identified Results of the Damage Parameters of the Beam

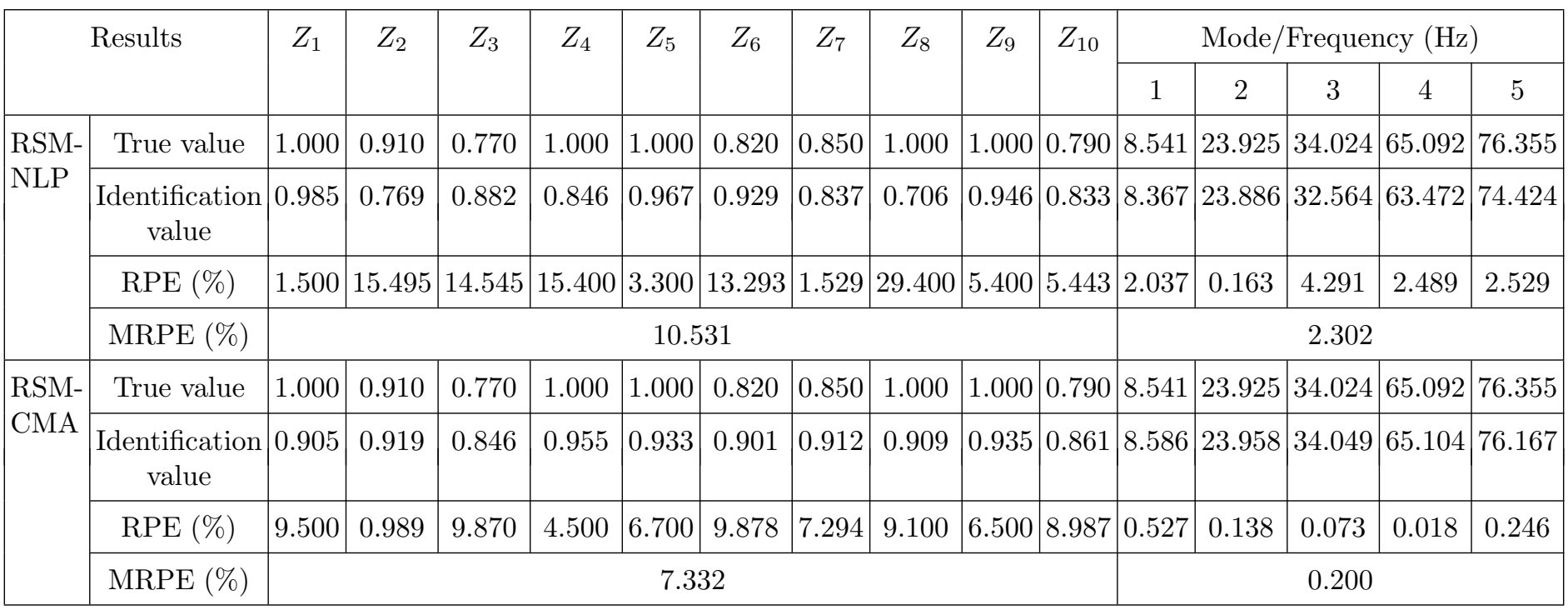

Note: $\mathrm{RPE}=(\mid$ True value-Identification value $\mid /$ True value $) \times 100 \%$. 
The hybrid method can accurately detect the damage location and degree of the structure.

The high detection confidence of the CMA combined with its insensitivity to number of parameters could lead to a promising damage detection procedure. Future research would involve experimental validation with real measurements from sensors.

\section{Acknowledgement}

This work was supported by the National Key Research and Development Program of China (2017YFC0806007), the National Science Fund for Distinguished Young Scholars (51425801), the National Natural Science Foundation of China (51808081), the Science and Technology Research Program of Chongqing Municipal Education Commission of China (KJQN201800701), the Science and Technology Research Project of Chongqing Science \& Technology Commission of China (cstc2017rgzn-zdyfX0029, cstc2018jscxmszdX0084), and the Science and Technology Project of Guizhou Provincial Transportation Department (2018122-013).

\section{References}

[1] X.G. Li, J.T. Zhou, L.Y. Zhang, et al., Automatic monitoring of continuous rigid frame bridges by a magneto-elastic effect method, International Journal of Robotics and Automation, 32(1), 2017, 41-47.

[2] J. Yang, J. Cheng, J.L. Wang, et al., Automatic deformation monitoring for large span bridge based on multi-constellation BDS and GPS system, International Journal of Robotics and Automation, 33(5), 2018, 534-541.

[3] J.Z. Xin, J.T. Zhou, S.X. Yang, et al., Bridge structure deformation prediction based on GNSS data using KalmanARIMA-GARCH model, Sensors (Basel), 18(1), 2018, 298.

[4] Z.H. Zong, J. Niu, and H. Wang, A review of structural damage identification methods based on the finite element model validation, China Civil Engineering Journal, 45(8), 2012, 121-130.

[5] C. Yang, X.B. Hou, L. Wang, et al., Applications of different criteria in structural damage identification based on natural frequency and static displacement, Science China Technological Sciences, 59(11), 2016, 1746.

[6] G. Li, R. Zhang, Y. Li, et al., Damage identification of bend-type structure based on modal flexibility-curvature and difference, Journal of Vibration Measurement and Diagnosis, 33(5), 2013, 902-906.

[7] A. Kaveh and A. Zolghadr, Guided modal strain energy-based approach for structural damage identification using tug-ofwar optimization algorithm, Journal of Computing in Civil Engineering, 31(4), 2017, 04017016.

[8] B. Moaveni, X.F. He, J.P. Conte, et al., Damage identification of a composite beam using finite element model updating, Computer-Aided Civil and Infrastructure Engineering, 23(5), 2008, 339-359.

[9] R.V. Farahani and D. Penumadu, Damage identification of a full-scale five-girder bridge using time-series analysis of vibration data, Engineering Structures, 115, 2016, 129-139.

[10] J. Grabowska, M. Palacz, and M. Krawczuk, Damage identification by wavelet analysis, Mechanical Systems and Signal Processing, 22(7), 2008, 1623-1635.

[11] A. Kunwar, R. Jha, M. Whelan, et al., Damage detection in an experimental bridge model using Hilbert-Huang transform of transient vibrations, Structural Control and Health Monitoring, 20(1), 2013, 1-15.

[12] U. Dackermann, J.C. Li, and B.J. Samali, Dynamic-based damage identification using neural network ensembles and damage index method, Advances in Structural Engineering, 13(13), 2010, 1001-1016.
[13] H. Ebrahimian, R. Astroza, J.P. Conte, et al., Nonlinear finite element model updating for damage identification of civil structures using batch Bayesian estimation, Mechanical Systems and Signal Processing, 84, 2016, 194-222.

[14] B. Moaveni, J.P. Conte, and F.M. Hemez, Uncertainty and sensitivity analysis of damage identification results obtained using finite element model updating, Computer-Aided Civil and Infrastructure Engineering, 24(5), 2009, 320-334.

[15] K.K. Nair, A.S. Kiremidjian, and K.H. Law, Time series-based damage detection and localization algorithm with application to the ASCE benchmark structure, Journal of Sound and Vibration, 291, 2006, 349-368.

[16] O. Begambre and J.E. Laier, A hybrid particle swarm optimization - simplex algorithm (PSOS) for structural damage identification, Advances in Engineering Software, 40(9), 2009, 883-891.

[17] O. Abdeljaber, O. Avci, S. Kiranyaz, et al., Real-time vibrationbased structural damage detection using one-dimensional convolutional neural networks. Journal of Sound and Vibration, 388, 2017, 154-170.

[18] F. Shabbir and P. Omenzetter, Model updating using genetic algorithms with sequential niche technique, Engineering Structures, 120, 2016, 66-182.

[19] H. Sun, H. Lus, and R. Betti, Identification of structural models using a modified artificial Bee Colony algorithm, Computers and Structures, 116, 2013, 59-74.

[20] H.J. Xu, J.K. Liu, and Z.R. Lu, Structural damage identification based on cuckoo search algorithm, Advances in Structural Engineering, 19(5), 2016, 849-859

[21] R.Q. Zhao and W.S. Tang, Monkey algorithm for global numerical optimization, Journal of Uncertain Systems, 2, 2008, 165-176.

[22] T.H. Yi, H.N. Li, G. Song, et al., Optimal sensor placement for health monitoring of high-rise structure using adaptive monkey algorithm, Structural Control and Health Monitoring, 22(4), 2015, 667-681.

[23] C.M. Ituarte-Villarreal, N. Lopez, and J.F. Espiritu, Using the monkey algorithm for hybrid power systems optimization, Procedia Computer Science, 12, 2012, 344-349.

[24] J.R. Wang, Y.X. Yu, Y. Zeng, et al., Discrete monkey algorithm and its application in transmission network expansion planning, Journal of Tianjin University, 43(9), 2010, 1-5.

[25] Z.R. Peng, H. Yin, A. Pan, et al., Chaotic monkey algorithm based optimal sensor placement, International Journal of Control and Automation, 9(1), 2016, 423-434.

[26] L.T. Stutz, I.C.S.S. Rangel, L.S. Rangel, et al., D.C. Knuppa structural damage identification built on a response surface model and the flexibility matrix, Journal of Sound and Vibration, 434, 2018, 284-297.

[27] X. Li, X.B. Li, and Y.H. Su, A hybrid approach combining uniform design and support vector machine to probabilistic tunnel stability assessment, Structural Safety, 61, 2016, 22-42.

\section{Biographies}

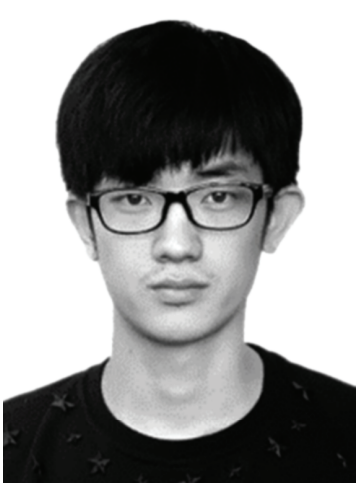

Jingzhou Xin is studying for a doctor's degree at Chongqing Jiaotong University, mainly engaged in bridge health monitoring. 


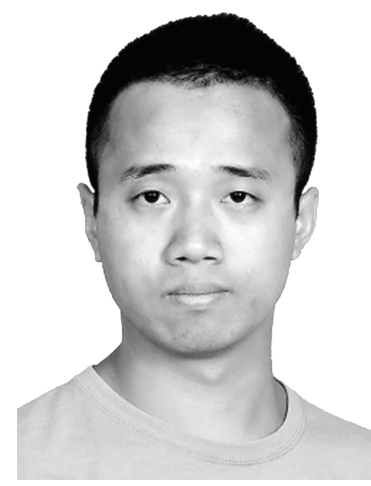

Hong Zhang is a lecturer of Chongqing Jiaotong University. His research interests include system integration, structural health monitoring, and non-destructive inspection.

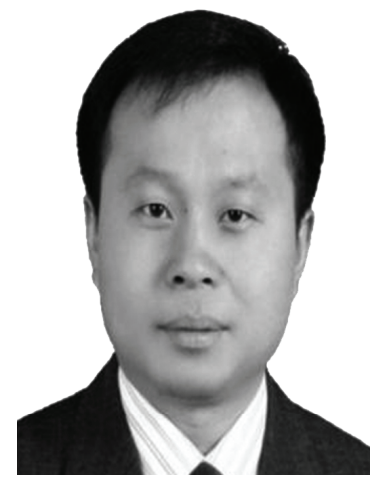

Jianting Zhou is a professor of Chongqing Jiaotong University. His research interests include bridge health monitoring and structural safety assessment.

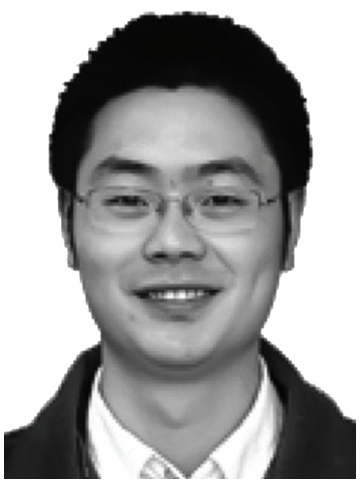

Xiaogang $L i$ is a senior engineer at T.Y. Lin International Engineering Consulting (China) Co., Ltd. His main research interests include bridge construction control and health monitoring.

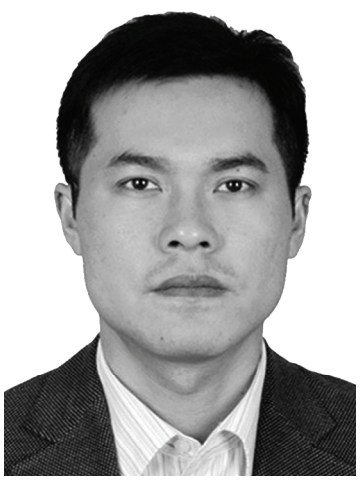

$H u M a$ works at Chongqing rail transit (Group) Co., Ltd. now. His main research interests include bridge construction control and health monitoring. 